\title{
Parity Anomaly Cancellation in Three-Dimensional QED with a Single Massless Dirac Fermion
}

\author{
Nikhil Karthik ${ }^{1, *}$ and Rajamani Narayanan ${ }^{2, \dagger}$ \\ ${ }^{1}$ Physics Department, Brookhaven National Laboratory, Upton, New York 11973-5000, USA \\ ${ }^{2}$ Department of Physics, Florida International University, Miami, Florida 33199, USA
}

(Received 23 March 2018; revised manuscript received 19 May 2018; published 26 July 2018)

\begin{abstract}
We study three-dimensional noncompact QED with a single two-component massless fermion and two infinitely massive regulator fermions of half the charge using the lattice overlap formalism. The parity anomaly is expected to cancel exactly between the massless and regulator fermions in the continuum, but this cancellation is inexact on a lattice akin to lattice chiral gauge theories. We show nonperturbatively that parity-breaking terms vanish in the continuum limit at any finite volume. We present numerical evidence that the resulting parity-invariant theory spontaneously breaks parity in the infinite volume limit.
\end{abstract}

DOI: 10.1103/PhysRevLett.121.041602

Introduction.-The standard model of particle physics is anomaly free due to an exact nontrivial cancellation of gauge anomalies [1] from different representations to all orders of perturbation theory. Chiral anomalies outside perturbation theory can be discussed geometrically [2] and the relation between consistent and covariant currents [3] plays a central role. Such fundamental issues should be addressed in any nonperturbative formalism of chiral gauge theories. The overlap formalism of chiral gauge theories on the lattice [4] was motivated [5] by an attempt to regularize a specific chiral gauge theory using an infinite number of Pauli-Villars fields [6] and the ability to use domain walls to create a chiral zero mode [7]. In order to discuss the problem of chiral anomalies in a gauge covariant and geometric manner, a two-form in the space of gauge fields defined through the curl of the difference between the covariant and consistent currents was introduced within the overlap formalism in Ref. [8], and it was identified to be Berry's curvature. Two sources contribute to this Berry's curvature for a chiral fermion in an anomalous representation - the first is due to the genuine continuum gauge anomaly that cannot be removed, and the second is due to the spatial smearing of the anomalous contribution due to finite lattice spacing. There is just the contribution due to smearing in an anomaly free chiral theory which can only be removed by fine-tuning the irrelevant terms in fermion action on the lattice [8]. The exceptions to the finetuning are QCD-like vector theories where the anomaly cancellation is trivial.

Published by the American Physical Society under the terms of the Creative Commons Attribution 4.0 International license. Further distribution of this work must maintain attribution to the author(s) and the published article's title, journal citation, and DOI. Funded by SCOAP ${ }^{3}$.
The odd-dimensional analog to chiral anomalies is parity anomaly [9-12] and this also can be discussed geometrically [13]. In this Letter, we consider a three-dimensional analog to the chiral gauge theories, where there is a nontrivial cancellation of parity anomaly between massless fermions and infinitely massive fermions, which is a property unique to three dimensions. The theory we consider is an Abelian $U(1)$ gauge theory with one massless Dirac fermion of charge $q$ and two infinitely massive fermions of charges $q / 2$ in a three-torus with physical size, $\ell^{3}$. This corresponds to the Euclidean continuum theory, with an implicit regularization,

$$
\mathcal{L}=\bar{\psi}(\not \partial+i q \not) \psi-\frac{q^{2} i}{8 \pi} \epsilon_{\mu \nu \rho} A_{\mu} \partial_{\nu} A_{\rho}+\frac{1}{4} F_{\mu \nu} F^{\mu \nu}
$$

written in standard notation in units where the coupling constant $g^{2}=1$. This theory has phenomenological relevance to the low-energy physics of fractional quantum Hall effect at half-filled Landau level [14-16]. Like in even dimensions, lattice regularization of this theory within the overlap formalism $[4,17,18]$ does not succeed in an exact cancellation of the parity anomaly. A salient result in this Letter is the numerical evidence for the restoration of parity invariance in the continuum at any finite physical volume without the need for fine-tuning the fermion action, which suggests a similar situation to hold in even dimensional chiral gauge theories as anticipated in Ref. [8]. This will also establish the existence of such three-dimensional theories outside perturbation theory. We will then present a numerical study of this theory in the infinite volume limit and provide evidence for spontaneous breaking of parity.

Modus operandi.-As is standard in lattice field theory, we discretize the physical volume $\ell^{3}$ using $L^{3}$ lattice points with the lattice spacing being $\ell / L$ [19]. The continuum 
limit is achieved by taking the $L \rightarrow \infty$ limit at fixed value of $\ell$. For the Abelian theory, the dynamical real lattice variables are $\theta_{\mu}(\mathbf{n})$ at the link connecting the lattice point at $\mathbf{n}$ to $\mathbf{n}+\hat{\mu}$. The lattice regularized partition function of the model in Eq. (1) using the overlap formalism $[17,18,20]$ is

$$
Z(\ell, L)=\int[d \theta] e^{-S_{g}(\theta)} \operatorname{det}\left(\frac{1+V_{\theta}}{2}\right) \operatorname{det}^{2} V_{\frac{1}{2} \theta}^{\dagger},
$$

where $S_{g}(\theta)$ is the noncompact gauge action on the lattice (obtained by discretizing the $F_{\mu \nu}^{2}$ term). The unitary operator $V_{q \theta}$ depends on the compact link variables $U_{\mu}^{q}(\mathbf{n})=e^{i q \theta_{\mu}(\mathbf{n})}$ where $q$ is the charge of the fermion coupled to the gauge field. We have set $q=1$ in Eq. (1) and the first determinant factor realizes the effective action obtained by integrating out the massless fermion in Eq. (1) and the second determinant factor realizes the ChernSimons term in Eq. (1) as induced by an infinitely massive fermion.

If we define the induced action $2 \mathcal{A}_{q}$ from the infinite mass fermion via, $\operatorname{det} V_{q \theta} \equiv \exp \left(2 i q^{2} \mathcal{A}_{q}\right)$, then we expect $\mathcal{A}_{q}(\theta)$ to be independent of $q$ for smooth gauge fields $[11,12,21,22]$, and be the same as the level-one ChernSimons action. If we perform the Euclidean parity transformation, under which $V_{q \theta} \rightarrow V_{q \theta}^{\dagger}$, the path integral in Eq. (2) transforms to

$Z(\ell, L)=\int[d \theta] e^{-S_{g}(\theta)} \operatorname{det} \frac{1+V_{\theta}}{2} \operatorname{det}^{2} V_{\frac{1}{2} \theta}^{\dagger} e^{-2 i \mathcal{A}(\theta)}$,

where

$$
\mathcal{A}(\theta)=\mathcal{A}_{1}(\theta)-\mathcal{A}_{\frac{1}{2}}(\theta) .
$$

Parity anomaly cancellation in the continuum means that $2 \mathcal{A}=0$ or equivalently, $\mathcal{A}=n \pi$ for $n=0, \pm 1$ as $L \rightarrow \infty$. On the lattice, however, the nontrivial anomaly cancellation between two different charges will result in $2 \mathcal{A}(\theta)$ being zero only on classically smooth backgrounds. An ensemble of gauge field configurations on the lattice away from the continuum limit will not be smooth and we do not expect $2 \mathcal{A}(\theta)=0(\bmod 2 \pi)$, leading to

$\operatorname{det} \frac{1+V_{\theta}}{2} \operatorname{det}^{2} V_{\frac{1}{2} \theta}^{\dagger}=\left|\operatorname{det} \frac{1+V_{\theta}}{2}\right| e^{i \mathcal{A}(\theta)} ; \mathcal{A} \in(-\pi, \pi]$,

which forms the core of the problem addressed in this Letter.

Our strategy can be summarized as follows. Using the rational hybrid Monte Carlo (RHMC) [23-25] method, an algorithm based on molecular dynamics evolution, we numerically simulate the theory on the lattice using the positive definite measure

$$
p_{+}(\theta)=\left|\operatorname{det} \frac{1+V_{\theta}}{2}\right| e^{-S_{g}},
$$

and consider the phase $e^{i \mathcal{A}}$ to be part of the observables. Our first aim is to study the distribution of $\mathcal{A}$ generated at a given $\ell$ and $L$ and show that the distribution has a tendency to approach a delta function for all $\ell$ as we take $L \rightarrow \infty$. As the lattice spacing increases with $\ell$ in a range of numerically feasible values of $L$, we can only provide reasonable numerical evidence for parity anomaly cancellation over a limited but wide range of $\ell$. Our second aim in this Letter is to assume that parity anomaly cancellation holds for all values of $\ell$ and study the infrared physics of the model in Eq. (1) using $p_{+}(\theta)$ as the measure.

Anomaly cancellation.-Using the LAPACK subroutines [26], we determined the phase $\mathcal{A}(\theta)$. Figure 1 shows the distribution $P(\mathcal{A})$ of $\mathcal{A}(\theta)$ as sampled using $p_{+}(\theta)$ in three panels, top to bottom, for $\ell=4,32,200$, respectively. Within each panel for a fixed $\ell$, the different symbols correspond to different lattice spacings. Due to the parityinvariant measure $p_{+}(\theta)$, the distributions are almost symmetric with small deviations resulting from finite statistics. We notice from the $\ell=4$ and 32 panels that $P(\mathcal{A})$ gets sharper as one approaches the continuum limit $L \rightarrow \infty$. However, this approach of the width of the distribution to zero is hard to see in the $\ell=200$ panel, and it is understandable since the finest lattice spacing ( $L=16$ ) at $\ell=200$, where we were able to compute $\mathcal{A}$ is 5.4 times larger than the one at $\ell=32(L=14)$. By putting together the data for $\mathcal{A}$ from all $\ell$ and $L$, we now justify that the distributions at larger $\ell$ will indeed get sharper at prohibitively large values of $L$. Since one expects the remnant phase $\mathcal{A}$ to be a volume integral of local irrelevant terms, we show the variance per unit physical volume, $\ell^{-3} \operatorname{Var}(\mathcal{A})$, as a function of lattice spacing, $\ell / L$,

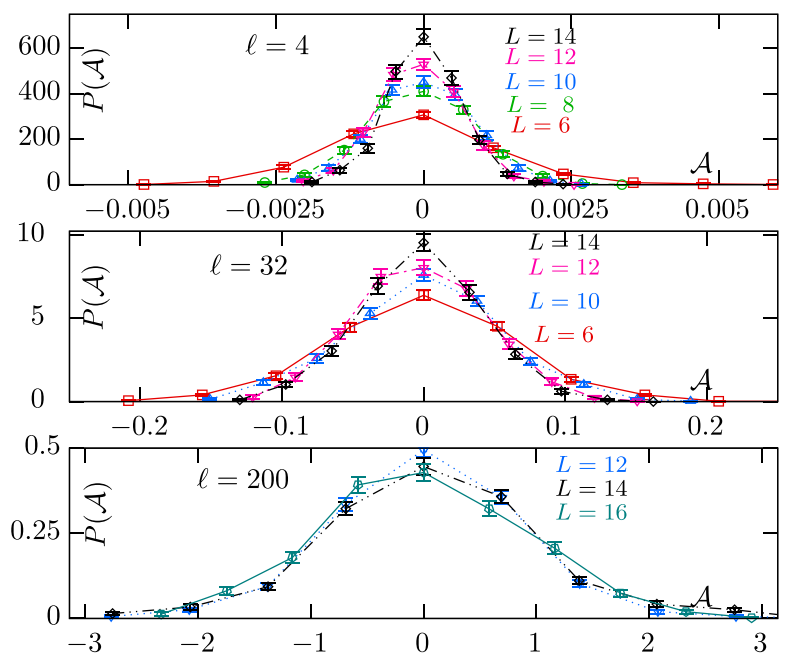

FIG. 1. The distributions of $\mathcal{A}(\theta)$ at different physical volumes $\ell^{3}$ are shown in the three panels. The different symbols correspond to different $L$. 


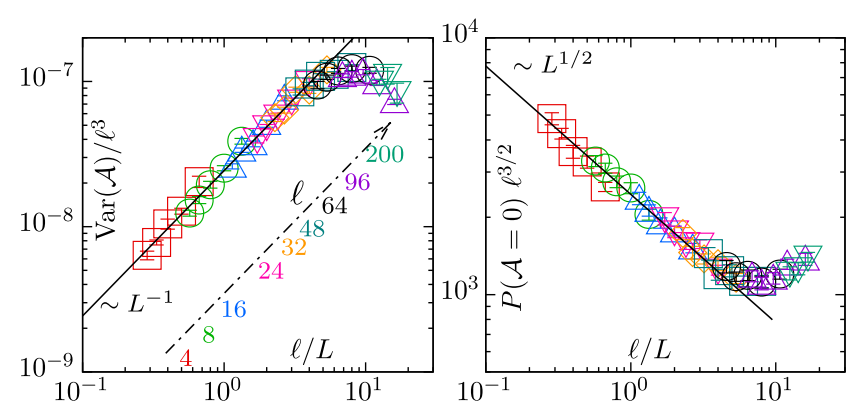

FIG. 2. The variance (left panel) and the height of the distribution at $\mathcal{A}=0$ (right panel) for $P(\mathcal{A})$, both scaled by appropriate powers of $\ell$, are shown as functions of lattice spacing.

in the left panel of Fig. 2. The data points of the same colored symbol belong to a fixed value of $\ell$ but differ in $L$, while different colored symbols correspond to different $\ell$ as specified near them. The data approximately falls on a universal curve, with $\operatorname{Var}(\mathcal{A}) \sim L^{-1}$ at smaller $\ell / L$. On the right panel of Fig. 2, we show the scaled peak height of the distribution, $\ell^{3 / 2} P(\mathcal{A}=0)$, as a function of $\ell / L$. The approximate data collapse suggests a $\sqrt{L}$ increase in the peak-height at smaller $\ell / L$. As expected, higher order effects in lattice spacing come into play in both figures for larger $\ell / L$. Based on these empirical observations, we find reasonable evidence for $P(\mathcal{A})$ to approach a delta function in the continuum limit at a fixed $\ell$ and it is important that one takes the continuum limit before taking the infinite volume limit.

We now discuss the sign of the fermion determinant. The distribution $P(\mathcal{A})$ on the coarser lattices, such as the one at $\ell=200$, covers the entire range $(-\pi, \pi]$, but still remains peaked at zero. Based on the arguments above, this implies that the distribution in the continuum limit will be peaked around zero, in spite of values of $\mathcal{A}$ close to $\pi$ being allowed in the essentially continuous molecular dynamics evolution of gauge fields used by the RHMC algorithm on coarser lattices. In principle, we could have found a separation of our ensemble into two sectors on coarser lattice spacings (corresponding to $\mathcal{A}(L=\infty)=0$ and $\pm \pi$ ) easily identified by a doubly peaked $P(\mathcal{A})$. In this case, it would have been necessary to have a zero of the fermion determinant along the RHMC's canonical evolution as the continuum limit is approached. Since we did not find this to be the case, our result is consistent with the absence of topological zero modes in odd-dimensional space without a boundary $[27,28]$. In this manner, we have succeeded in demonstrating that Eq. (2) has a parity invariant as well as an effectively positive measure in the continuum.

Another quantity relevant to the anomaly cancellation is

$$
J_{i}^{q}(\mathbf{n})=\frac{\delta}{\delta \theta_{i}(\mathbf{n})} \mathcal{A}_{q}(\theta)
$$

which is a fermion-induced pseudovector current in lattice units, and the expectation value of its magnitude is $E_{q}(\mathbf{n})=$ $\left\langle\mathbf{J}^{q}(\mathbf{n}) \cdot \mathbf{J}^{q}(\mathbf{n})\right\rangle_{+}$. One expects $J_{i}^{q}(\mathbf{n})$ to depend locally on the flux $\sim \epsilon_{i j k} \Delta_{j} \theta_{k}$, but need not be ultralocal and get smeared around $\mathbf{n}$ as discussed in Ref. [8]. In the absence of such an ultralocality, $E(\mathbf{n})=E_{1}(\mathbf{n})-E_{1 / 2}(\mathbf{n})$ will not vanish at finite lattice spacing but it must vanish faster than $E_{1}(\mathbf{n})$ and $E_{1 / 2}(\mathbf{n})$ as one approaches the continuum. In Fig. 3, we put together the data from all $\ell$ and $L$ for $E_{1}$ and $E_{1 / 2}$ at an arbitrarily chosen $\mathbf{n}$, and show it as a function of lattice spacing $\ell / L$. The data from different values of $\ell$ fall on the same curve due to the local nature of this observable. The lattice spacing scaling of $E_{1}$ and $E_{1 / 2}$ is $\ell / L$, the same as the average local energy density. With this combined data, we see that $E$ falls off with the lattice spacing like $(\ell / L)^{3}$, faster than $E_{1}$ or $E_{1 / 2}$ by two powers of lattice spacing, ensuring again that the theory will be parityinvariant at all values of $\ell$ studied here.

Having demonstrated the path integral measure is anomaly free in the continuum limit, it is also imperative that we show the vacuum expectation values of parity-odd observables vanish in the continuum limit. Decomposing any observable $\mathcal{O}$ into its parity-even and odd components $\mathcal{O}_{e}$ and $\mathcal{O}_{o}$, respectively, its expectation value can be written as

$\langle\mathcal{O}(\theta)\rangle=\frac{\left\langle\mathcal{O}_{e}(\theta) \cos \mathcal{A}(\theta)\right\rangle_{+}}{\langle\cos \mathcal{A}(\theta)\rangle_{+}}+i \frac{\left\langle\mathcal{O}_{o}(\theta) \sin \mathcal{A}(\theta)\right\rangle_{+}}{\langle\cos \mathcal{A}(\theta)\rangle_{+}}$.

We want to show that in the continuum limit, the parityeven first term on the right-hand side becomes $\left\langle\mathcal{O}_{e}\right\rangle_{+}$and the parity-odd second term vanishes. We consider the correction $C_{e}+i C_{o}=\langle\mathcal{O}\rangle-\left\langle\mathcal{O}_{e}\right\rangle_{+}$as a function of $L$. For $\mathcal{O}$, we used the dimensionless lowest positive eigenvalue $\lambda_{1}^{+}(\theta) \ell$ of the inverse of massless Hermitian overlap Dirac propagator, $i G^{-1}(\theta) L=i\left(1+V_{\theta} / 1-V_{\theta}\right) L$, at different $L$. In Fig. 4 , we show the decreasing behavior of both $C_{o}$ and $C_{e}$ at different fixed $\ell$, as $L$ is increased. The different colored symbols in the plot belong to different $\ell$. At any finite $L, C_{o}$ is significantly nonzero and indeed decreases when the lattice spacing is made smaller. On finer lattices, a distinct $L^{-\Delta}$ behavior with an empirical value

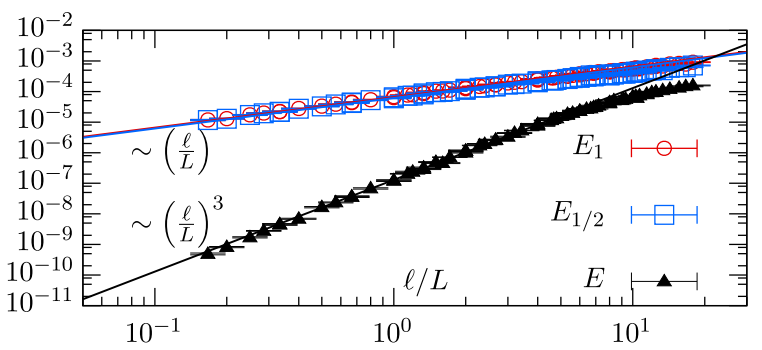

FIG. 3. The dependence of $E_{1}, E_{1 / 2}$ and their difference, $E$, on lattice spacing $\ell / L$. 

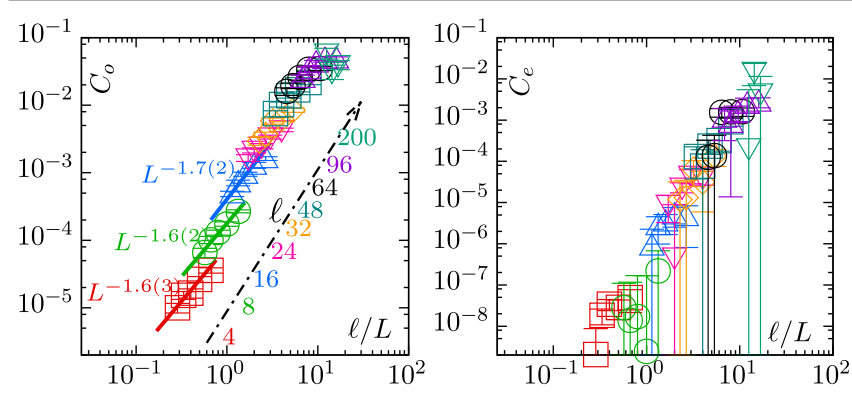

FIG. 4. The approach of $C_{o}$ (left) and $C_{e}$ (right) to 0 in the continuum limit.

$\Delta \approx 1.5$ is seen. For the data at larger $\ell / L$, a downward curvature is seen implying the asymptotic values of $\Delta$ will be greater than what can be extracted from the data (which is about 1.2). On the other hand, the ultraviolet physics of anomaly cancellation seems to decouple from the infrared parity-even expectation values, as seen from the fact that $C_{e}$ is much less than $0.1 \%$ of $\left\langle\frac{1}{2}\left[\lambda_{1}^{+}(\theta)+\lambda_{1}^{+}\left(\theta_{p}\right)\right] \ell\right\rangle_{+}$(and about 2 to 3 orders of magnitude lesser than the corresponding $C_{o}$ ) in the range of $\ell$ we studied. In fact, for $L>10, C_{e} \approx 0$ within a $1.5-\sigma$ error range.

Spontaneous symmetry breaking of parity.-Having numerically established a parity-invariant theory with a positive measure in a certain range of $\ell$ that was numerically accessible, we will assume this to be the case for higher values of $\ell$ and study the infrared behavior of the theory by taking the $\ell \rightarrow \infty$ limit using the $p_{+}(\theta)$ measure. A possibility is the spontaneous symmetry breaking (SSB) of parity leading to a nonzero bilinear condensate $\Sigma$, i.e., at finite fermion mass $m$ and infinite volume, $\langle\bar{\psi} \psi\rangle(m)=\Sigma(m /|m|)+O(m)$. To study this, we focus on the discrete dimensionless Dirac operator eigenvalues ordered by magnitude, $0<\ell \lambda_{1}(\theta ; \ell)<\ell \lambda_{2}(\theta ; \ell)<\ldots$, (which are technically obtained from $L\left|G^{-1}(\theta)\right|$ ), at finite $\ell$. We first take the $L \rightarrow \infty$ continuum limit of $\left\langle\lambda_{i}(\ell) \ell\right\rangle$ (using $L$ from 12 to 24 ) at different fixed $\ell$ ranging from 4 to 250 for this study before considering the $\ell \rightarrow \infty$ limit.

The probability distribution of $\lambda_{i}(\theta ; \ell)$, as sampled in the Monte Carlo calculation, will exhibit several well separated peaks consistent with a spectrum that is discrete. Perturbation theory will hold as $\ell \rightarrow 0$ and $\left\langle\lambda_{i}(\ell)\right\rangle$ will be proportional to $\ell^{-1}$. If the theory spontaneously breaks parity as $\ell \rightarrow \infty$, then $\left\langle\lambda_{i}(\ell)\right\rangle \sim \ell^{-3}$ (due to a finite eigenvalue density near zero [29]) and in addition, the distributions of the individual eigenvalues should also match with those from an appropriate random matrix theory (RMT) ensemble [30-32]. If we define $\Sigma_{i}(\ell)$ through the means $\left\langle\lambda_{i}(\ell)\right\rangle$ and $z_{i}$ of the two respective distributions,

$$
\left\langle\lambda_{i}(\ell)\right\rangle \Sigma_{i}(\ell) \ell^{3}=z_{i},
$$

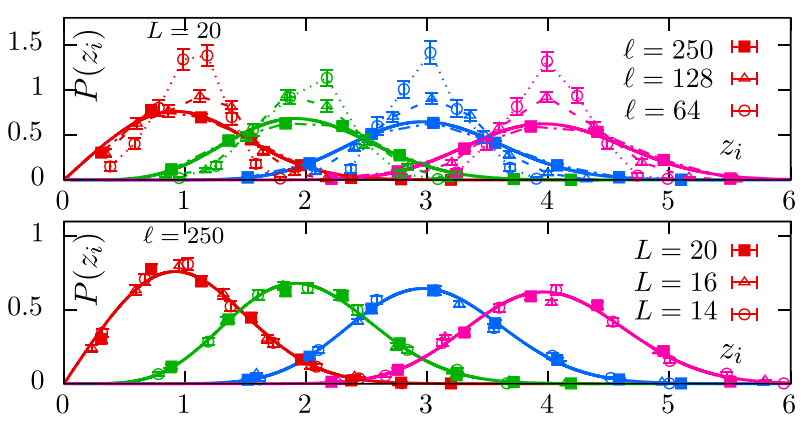

FIG. 5. Comparison of the distributions of $\lambda_{i} \Sigma_{i}(\ell) \ell^{3}$ (symbols connected by dotted lines), and the RMT eigenvalues $z_{i}$ (solid curves). The red, green, blue, and purple curves correspond to $i=1,2,3$, 4, respectively. Top: Volume dependence at fixed $L=20$. Bottom: Lattice spacing dependence at $\ell=250$.

then $\Sigma_{i}(\ell)$ for different $i$ should approach the same nonzero value $\Sigma$ (the value of the condensate) as $\ell \rightarrow \infty$.

Figure 5 shows a comparison of the distributions of the scaled, four low-lying Dirac eigenvalues, $\ell^{3} \Sigma_{i}(\ell, L)$ $\lambda_{i}(\theta ; \ell, L)$, to the distributions from the RMT, which are shown as solid curves in the plots. The top panel shows the volume dependence of the distributions at a fixed number of lattice points $L=20$. One can see the distributions approaching the RMT as $\ell$ is increased from $\ell=64$ to $\ell=250$. The bottom panel shows this agreement between the Dirac and RMT eigenvalue distributions at $\ell=250$ is robust as the number of lattice points $L$ is made larger from $L=14$ to 20 . A quantitative estimate shows that the deviation of the data from the RMT distributions becomes smaller with increasing $\ell$ and approaches zero in the infinite volume limit. This agreement with RMT shows the presence of SSB.

Figure 6 shows $\Sigma_{i}(\ell)$ as extracted from the matching with RMT using Eq. (9), as a function of $\ell$. The different symbols are the values of $\Sigma_{i}(\ell) ; i=1,2,3,4$ in the continuum at different fixed $\ell$. At any finite $\ell$ the values of $\Sigma_{i}(\ell)$ from different $i$ do not agree, as expected. Assuming the existence of the finite nonzero value of the condensate $\Sigma$ in the infinite volume limit, we used $\Sigma_{i}(\ell)=\Sigma_{i}+k_{1} \ell^{-1}+k_{2} \ell^{-2}$, to fit the entire range of finite

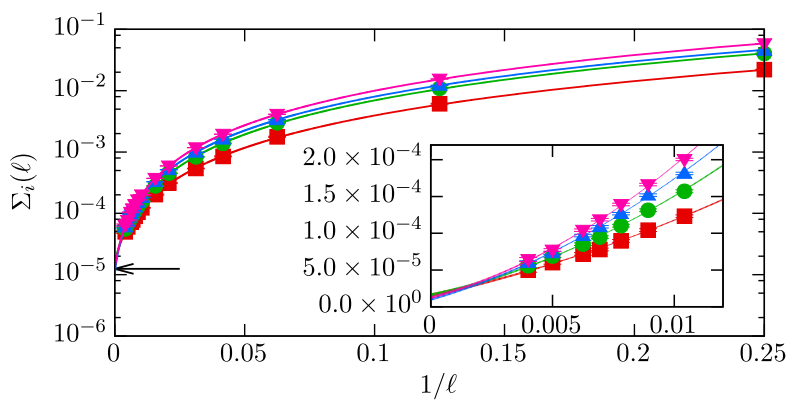

FIG. 6. The infinite volume extrapolation of $\Sigma_{i}(\ell)$. The red, green, blue, and purple points and curves correspond to $i=1,2$, 3,4 , respectively. 
$\ell$ data. These fits are shown by the curves. The inset magnifies the large $\ell$ region. We find the extrapolated values of $\Sigma_{i} \times 10^{5}$ from $i=1,2,3,4$ to be 1.5(3),1.5(3), $1.0(2)$, and 1.2(2), respectively. Though the extrapolated values are about factor 5 smaller than the available data point, the agreement between different extrapolated values of $\Sigma_{i}$, together with the remarkable agreement with RMT distributions are indications of a unique $\Sigma \neq 0$ at infinite $\ell$.

Discussion.-An earlier attempt [33] to verify the cancellation of anomalies in a two-dimensional chiral gauge theory by directly establishing gauge invariance in the continuum suffered from the fact that there is no concept of smooth gauge transformations in the continuum limit. In light of the results in this Letter, it would be interesting to revisit this problem by a computation of the continuum limit of the Berry's curvature [8] in a sequence of lattice gauge field ensembles at different lattice spacings. Of experimental relevance are the response functions of the single flavor theory studied here with the topological current coupled to a background compact gauge field $\phi$, which can be realized in our lattice setup by including the term $\operatorname{det}\left[V_{\theta-\phi} V_{\theta}^{\dagger} V_{\phi}^{\dagger}\right]$ in Eq. (2). This particular model appears in recent discussions of duality between fermion theories [34-36]. It would be interesting to see if the SSB has any effect on the induced action for $\phi$. It is trivial to extend the overlap formalism presented here for threedimensional QED with an arbitrary number of flavors $(N)$ of massless Dirac fermions and arbitrary number of flavors $(k)$ of infinite mass fermions. This is a numerical challenge that could benefit from the various approaches developed for the sign problem in finite density QCD.

The authors would like to thank Jac Verbaarschot for a discussion on the RMT kernel. This work used the Extreme Science and Engineering Discovery Environment (XSEDE), which is supported by NSF under Grant No. ACI-1548562. Resources at Pittsburgh Supercomputing Center, San Diego Supercomputer Center, LSU Center for Computation and Technology, and at University of Texas at Austin were used under the XSEDE allocation TG-PHY170011. Some computations in this Letter were also performed on JLAB computing clusters under a USQCD type $C$ project. R. N. acknowledges partial support by the NSF under Grant No. PHY-1515446. N. K. acknowledges support by the U.S. Department of Energy under Contract No. DESC0012704.

"nkarthik@bnl.gov

†rajamani.narayanan@fiu.edu

[1] M. D. Schwartz, Quantum Field Theory and the Standard Model (Cambridge University Press, Cambridge, England, 2014).

[2] L. Alvarez-Gaume and P. H. Ginsparg, Nucl. Phys. B243, 449 (1984).

[3] W. A. Bardeen and B. Zumino, Nucl. Phys. B244, 421 (1984).
[4] R. Narayanan and H. Neuberger, Nucl. Phys. B443, 305 (1995).

[5] R. Narayanan and H. Neuberger, Phys. Lett. B 302, 62 (1993).

[6] S. A. Frolov and A. A. Slavnov, Phys. Lett. B 309, 344 (1993).

[7] D. B. Kaplan, Phys. Lett. B 288, 342 (1992).

[8] H. Neuberger, Phys. Rev. D 59, 085006 (1999).

[9] S. Deser, R. Jackiw, and S. Templeton, Ann. Phys. (N.Y.) 140, 372 (1982).

[10] S. Deser, R. Jackiw, and S. Templeton, Phys. Rev. Lett. 48, 975 (1982).

[11] A. J. Niemi and G. W. Semenoff, Phys. Rev. Lett. 51, 2077 (1983).

[12] A. N. Redlich, Phys. Rev. D 29, 2366 (1984).

[13] L. Alvarez-Gaume, S. Della Pietra, and G. W. Moore, Ann. Phys. (N.Y.) 163, 288 (1985).

[14] D. T. Son, Phys. Rev. X 5, 031027 (2015).

[15] M. A. Metlitski and A. Vishwanath, Phys. Rev. B 93, 245151 (2016).

[16] C. Wang and T. Senthil, Phys. Rev. B 93, 085110 (2016).

[17] Y. Kikukawa and H. Neuberger, Nucl. Phys. B513, 735 (1998).

[18] R. Narayanan and J. Nishimura, Nucl. Phys. B508, 371 (1997).

[19] See Supplemental Material at http://link.aps.org/ supplemental/10.1103/PhysRevLett.121.041602, for details of the numerical computations and analysis are provided.

[20] N. Karthik and R. Narayanan, Phys. Rev. D 94, 065026 (2016).

[21] A. Coste and M. Luscher, Nucl. Phys. B323, 631 (1989).

[22] N. Karthik and R. Narayanan, Phys. Rev. D 92, 025003 (2015).

[23] A. D. Kennedy, I. Horvath, and S. Sint, Nucl. Phys. B, Proc. Suppl. 73, 834 (1999).

[24] M. A. Clark and A. D. Kennedy, Nucl. Phys. B, Proc. Suppl. 129-130, 850 (2004).

[25] S. Duane, A. Kennedy, B. Pendleton, and D. Roweth, Phys. Lett. B 195, 216 (1987).

[26] E. Anderson, Z. Bai, C. Bischof, S. Blackford, J. Demmel, J. Dongarra, J. Du Croz, A. Greenbaum, S. Hammarling, A. McKenney, and D. Sorensen, LAPACK Users' Guide, 3rd ed. (Society for Industrial and Applied Mathematics, Philadelphia, 1999).

[27] M. F. Atiyah and I. M. Singer, Bull. Am. Math. Soc. 69, 422 (1963).

[28] C. Callias, Commun. Math. Phys. 62, 213 (1978).

[29] T. Banks and A. Casher, Nucl. Phys. B169, 103 (1980).

[30] J. J. M. Verbaarschot and I. Zahed, Phys. Rev. Lett. 73, 2288 (1994).

[31] P. H. Damgaard and S. M. Nishigaki, Phys. Rev. D 57, 5299 (1998).

[32] S. M. Nishigaki, Proc. Sci. LATTICE2015 (2016) 057.

[33] R. Narayanan and H. Neuberger, Nucl. Phys. B477, 521 (1996).

[34] D. F. Mross, J. Alicea, and O. I. Motrunich, Phys. Rev. Lett. 117, 016802 (2016).

[35] M. Cheng and C. Xu, Phys. Rev. B 94, 214415 (2016).

[36] C. Cordova, P.-S. Hsin, and N. Seiberg, arXiv:1712.08639. 